\title{
FS5 sun exposure survivability analysis
}

\author{
Ming-Ying Hsu *, Shenq-Tsong Chang, and Ting-Ming Huang \\ Instrument Technology Research Centre, National Applied Research Laboratories, HsinChu, Taiwan
}

\author{
Article history: \\ Received 24 December 2015 \\ Revised 12 June 2016 \\ Accepted 17 June 2016 \\ Keywords: \\ RSI, Radiometry, Radiance, ASH \\ Citation: \\ Hsu, M. Y., S. T. Chang, and T. M. \\ Huang, 2017: FS5 sun exposure \\ survivability analysis. Terr. Atmos. \\ Ocean. Sci., 28, 149-156, doi: \\ 10.3319/TAO.2016.06.17.01(EOF5)
}

\begin{abstract}
During the Acquisition and Safe Hold (ASH) mode, FORMOAT-5 (FS5) satellite attitude is not fully controlled. Direct sun exposure on the Remote Sensing Instrument (RSI) satellite telescope sensor may occur. The sun exposure effect on RSI sensor performance is investigated to evaluate the instrument's survivability in orbit. Both satellite spin speed and sun exposure duration are considered as the key parameters in this study. A simple radiometry technique is used to calculate the total sun radiance exposure to examine the RSI sensor integrity. Total sun irradiance on the sensor is computed by considering the spectral variation effect through the RSI's five-band filter. Experiments that directly expose the sensor to the sun on the ground were performed with no obvious performance degradation found. Based on both the analysis and experiment results, it is concluded that the FS5 RSI sensor can survive direct sun exposure during the ASH mode.
\end{abstract}

\section{INTRODUCTION}

A remote sensing satellite is usually equipped with a telescope to effectively collect target signals. The telescope must be designed to a set of required parameters such as signal-to-noise ratio, optimized F-number and corresponding integration time, etc. During normal operation, an Earth observation satellite is in earth-pointing mode so that the ground target can be imaged without directly exposing the image sensor to sunlight. It takes time to bring a tumbling satellite into a safe and controlled attitude right after separating from the launch vehicle. The sensitive image sensor of such a satellite could be damaged or degraded by direct exposure to sunlight.

Possible space radiation damage to the ESA's Gaia satellite CCDs was modelled and analysed to determine an overall radiation calibration strategy (Seabroke et al. 2008). The radiometric issue can be managed by using a sensor shutter in front of the telescope as a protective shield or a shutter wheel as that designed for the modern Landsat satellites (Irons et al. 2012).

FORMOAT-5 (FS5) will operate in a sun synchronous orbit of $720 \mathrm{~km}$ altitude with similar radiometric issues. As the primary payload, Remote Sensing Instrument (RSI)

\footnotetext{
* Corresponding author

E-mail:myhsu@itrc.narl.org.tw
}

optical sensor using a CMOS (complementary metal-oxidesemiconductor) technology is composed of a 12000-pixel Panchromatic (PAN) band and four 6000-pixel Multi-Spectral (MS) bands. The pixel size for the PAN band is 10 and $20 \mu \mathrm{m}$ for the MS band. The RSI telescope has a focal length of $3600 \mathrm{~mm}$, primary mirror of $450 \mathrm{~mm}$ diameter, and Fnumber of 8 . The field of view (FOV) of the RSI sensor is $1.91^{\circ} \times 1.4^{\circ}$. A five stripe band-pass filter is mounted on top of the same substrate, as shown in Fig. 1. The Panchromatic spectral ranges $(450-700 \mathrm{~nm}), \mathrm{B} 1(455-515 \mathrm{~nm}), \mathrm{B} 2(525$ - $595 \mathrm{~nm}), \mathrm{B} 3(630-690 \mathrm{~nm})$, and B4 (763 - $898 \mathrm{~nm})$ are shown in Fig. 2 (Hsiao et al. 2010). Since no special protection (i.e., shutter) has been designed for protecting the FS5 CMOS sensor, detailed analysis of the sun irradiance effect on the sensor performance becomes essential. Potential direct sun exposure due to the satellite tumbling during the Acquisition and Safe Hold (ASH) mode is also taken into account in this study.

\section{METHODOLOGY}

The sun exposure effect on the FS5 optical sensor is analysed following the radiometry calculations described by Dereniak and Boreman (1996) and Palmer and Grant (2009). The transmittance of the five stripe band-pass filter, especially at the short-wave infrared range, is also inspected 


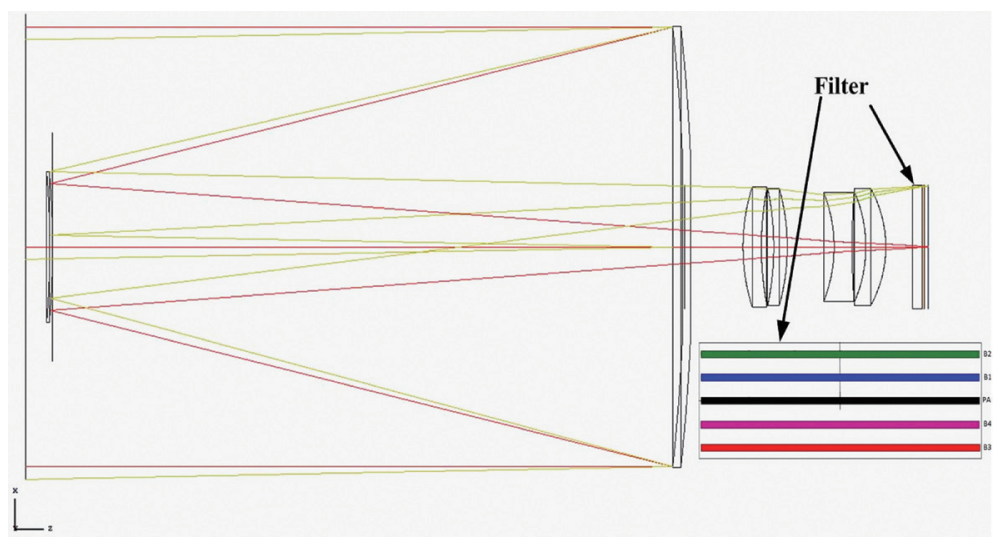

Fig. 1. RSI optical design profile. (Color online only)

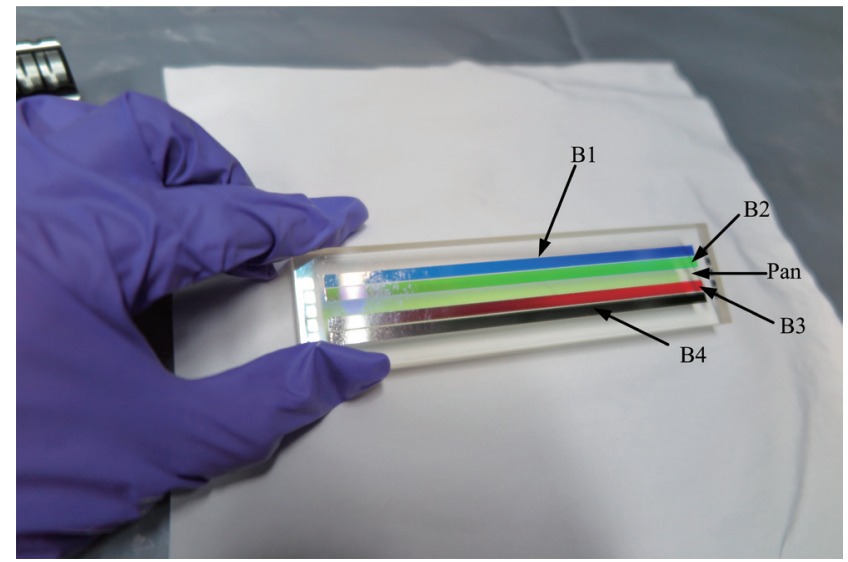

Fig. 2. RSI band pass filter. (Color online only)

in detail and taken into consideration. The irradiance contribution due to the short-wave infrared range is also included in this analysis. The worst radiometry case collected by this sensor is the RSI telescope aimed directly at the sun when the sun travels across the sensor focal plane at $0.085^{\circ} \mathrm{sec}^{-1}$ satellite spin speed given by the FS5 design group.

The sensor detector orientation, which collects the most sun power is the worst case defined by considering a two-dimensional relation, as shown in Fig. 3. The sun angle at the earth, $\theta_{\text {sun }}$, can be calculated using Eq. (1),

$\theta_{\text {sun }}=\tan ^{-1}\left(\frac{R_{\text {sun }}}{D_{S \& E}}\right) \times 2$

Where, $R_{\text {sun }}$ is the radius of the sun $\left(6.955 \times 10^{5} \mathrm{~km}\right)$ and $D_{S \& E}$ is the distance between the sun and earth $\left(1.47 \times 10^{8} \mathrm{~km}\right)$. The satellite FOV can be calculated using Eq. (2)

$\theta_{F O V}=\tan ^{-1}\left(\frac{D_{\text {swath }} \times 0.5}{D_{\text {orbit }}}\right) \times 2$
Where, $D_{\text {swath }}$ is the swath width of FS5 RSI $(24 \mathrm{~km})$ and $D_{\text {orbit }}$ is the satellite's orbit altitude $(720 \mathrm{~km})$. The maximum exposure time to the sun, $T_{\max }$, is defined using Eq. (3)

$T_{M a x}=\frac{\left|\theta_{\text {sun }}+\theta_{F O V}\right|}{\omega}$

Where, $\omega$ is the angular velocity of the satellite $\left(0.085^{\circ} \mathrm{sec}^{-1}\right)$. The time period of exposure to the maximum amount of the sun's energy, $T_{S E S}$, is calculated using Eq. (4)

$T_{S E S}=\frac{\left|\theta_{\text {sun }}-\theta_{F O V}\right|}{\omega}$

The solid angle, $\Omega_{\text {sun }}$, when the sun subtends at the earth can be calculated using

$\Omega_{\text {sun }}=\frac{\pi \times R_{\text {sun }}^{2}}{D_{S \& E}^{2}}$ 

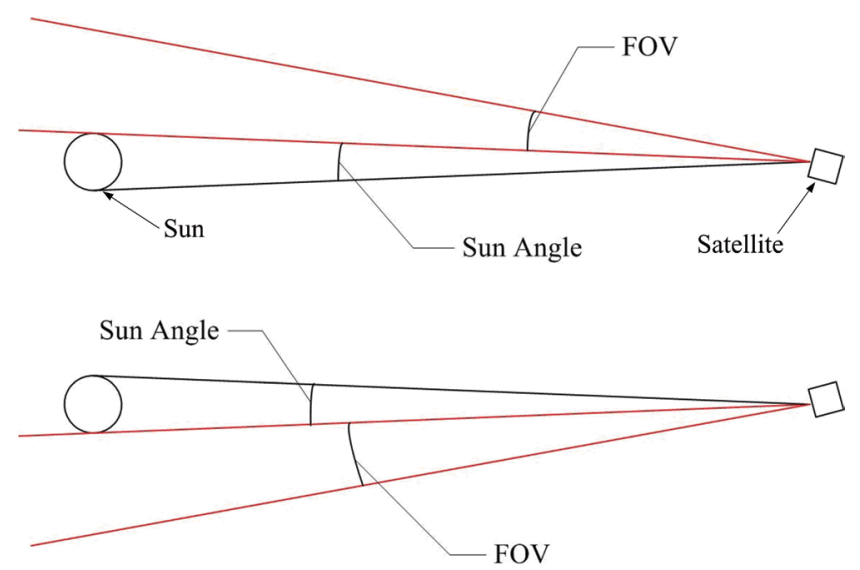

Fig. 3. Illustration of RSI field of view (FOV) and sun angle. (Color online only)

The sun's radiance, $L$, is calculated using Eq. (6)

$$
L=\frac{\phi_{d}}{A_{\text {earth }} \times \Omega_{\text {sun }}}
$$

Where, $\phi_{d} / A_{\text {earth }}$ is the solar constant $\left(1368 \mathrm{~W} \mathrm{~m}^{-2}\right)$ since there is little atmospheric absorption on the orbit, $\phi_{d}$ is the radiant flux. This value can then be used in Eq. (7) to determine the solar irradiance at the sensor surface

$E_{F P A}=L \times \frac{A_{\text {lens }}}{f^{2}}$

Where, $A_{\text {lens }}=0.116 \mathrm{~m}^{2}$ is the lens aperture area and $f=3.6 \mathrm{~m}$ is the effective focal length.

For the worst case, the satellite's FOV rotates through the sun such that the sensor collects the most power during sun exposure. The total sun energy exposure at the sensor is calculated using Eq. (8)

$E_{F P A}^{\prime}=L \times \frac{A_{\text {lens }}}{f^{2}} \times \cos \theta$

Where, $E_{F P A}^{\prime}$ is the energy and $\cos \theta \operatorname{compensates~for~the~}$ satellite rotation angle. The $\theta$ is the angle between FOV and the solar angle; $\cos \theta=1$ when the satellite rotation FOV covers the solar angle. Solar irradiance, from the visible to the near-infrared range, is typically shown in Fig. 4. The analysis above does not consider the band-pass filter effect. Radiance contributed by certain spectral range on a sensor detector can be calculated using Eq. (9) without considering the effect of a telescope.

$$
L=\int_{\lambda_{1}}^{\lambda_{2}} L_{S}(\lambda) d \lambda
$$

Where $L_{S}(\lambda)$ is the sun radiance at spectral wave length $\lambda$; $\lambda_{1}$ and $\lambda_{2}$ stand for the spectral range in which the total radiance, $L$, is calculated.

\section{RESULTS}

\subsection{Solar Energy Calculation}

Using FS5 RSI's design data and the equations as defined in section 2, one can calculate the key parameters for the FS5 optical sensor. The sun view angle at the earth, $\theta_{\text {sun }}$, is $0.542^{\circ}$ and the satellite FOV, $\theta_{F O V}$, is $1.91^{\circ}$. The maximum exposure time, $T_{\max }$, is $28.85 \mathrm{sec}$ and the exposure duration, $T_{S E S}$, to the maximum amount of sun energy is 16.1 sec. In addition, the solid angle of the sun, $\Omega_{\text {sun }}$, is $7.022 \times$ $10^{-5} \mathrm{sr}$, and the sun's radiance, $L$, is $1.948 \times 10^{7} \mathrm{~W} \mathrm{~m}^{-2} \cdot \mathrm{sr}$. Per Eq. (7) the total solar power from the sun to the RSI image sensor can be estimated as $1.744 \times 10^{5} \mathrm{~W} \mathrm{~m}^{-2}$.

The power collected during the worst case of direct sun exposure at the image sensor is calculated as shown in Fig. 5, based on the RSI focal plane area of $0.01056 \mathrm{~m}^{2}$. As expected, the sun power level reaching the focal plane is a function of the relative satellite spinning incident angle. The maximum sun power value is $1650 \mathrm{~W}$. The filter transmittance was designed according to the coating requirement for both visible and near-infrared band. The FS5 RSI filter transmittance data is shown in Fig. 6, which indicates the irradiance contribution from the short-wave infrared band is significant and should be included in the study.

\subsection{Solar Irradiance at Visible through Near-Infrared Band}

When the sun light goes through the RSI five-band filter to reach the image sensor, sun irradiance collected by the sensor can be calculated using Eq. (9) without considering the telescope structure influence. The total irradiance for the spectral range from $350-2500 \mathrm{~nm}$ was found to be 


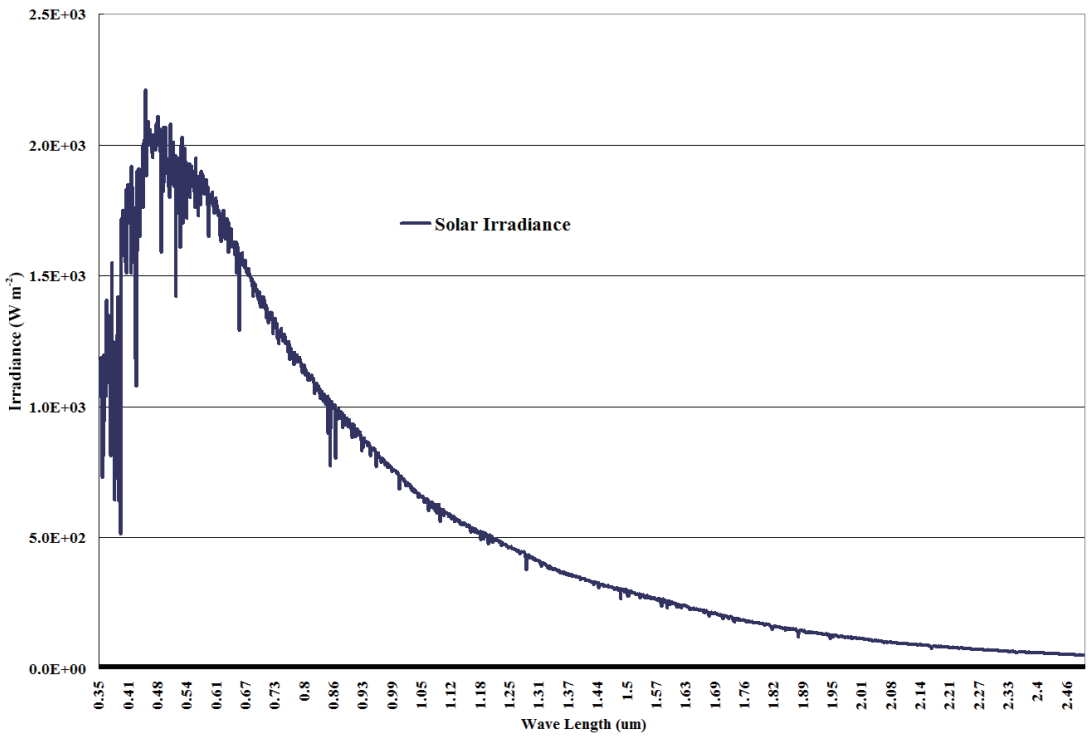

Fig. 4. Solar irradiance profile detected on the top of earth atmosphere. (Color online only)

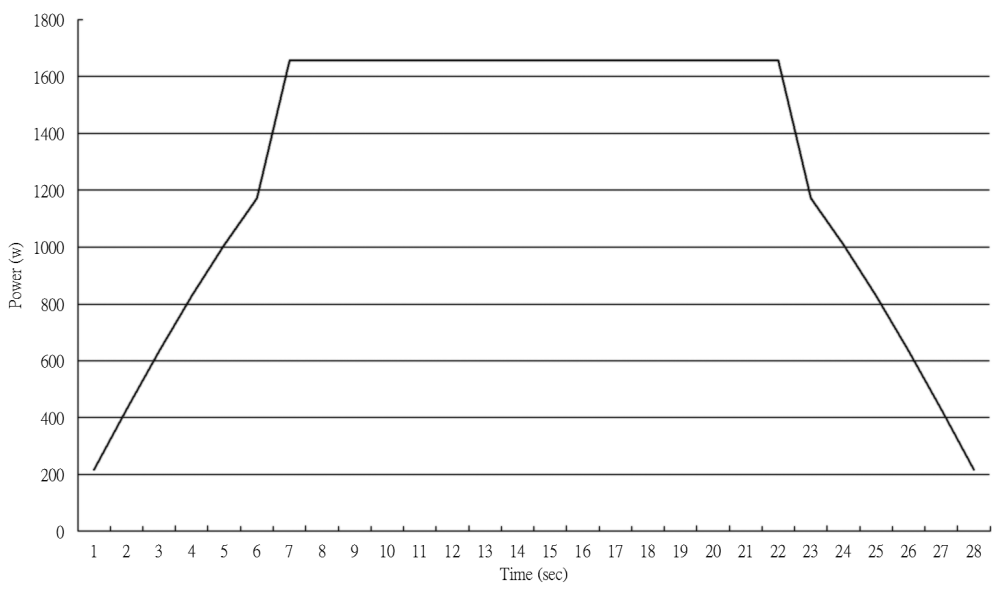

Fig. 5. Solar irradiance (power) level during sun exposure period.

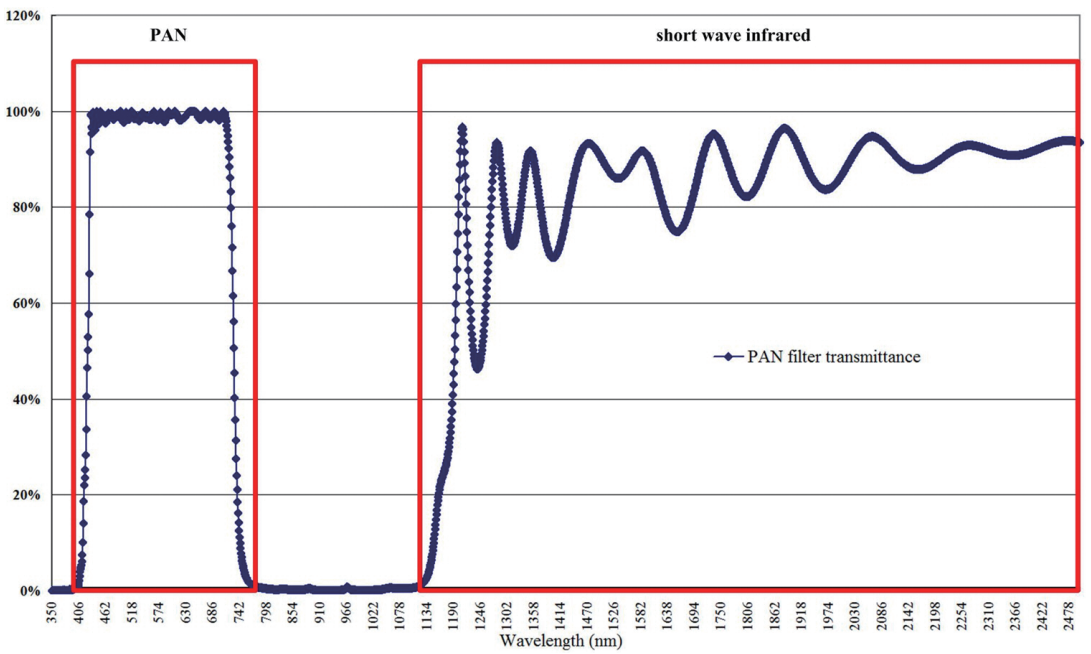

Fig. 6. FS5 filter transmittance at visible through short-wave infrared bands. (Color online only) 
$1.27 \times 10^{3} \mathrm{~W} \mathrm{~m}^{-2}$ without considering the filter influence. When the band-pass filter effect is considered, the irradiance through the PAN band filter from 450 - $700 \mathrm{~nm}$ becomes $4.46 \times 10^{2} \mathrm{~W} \mathrm{~m}^{-2}$, which is about $35.08 \%$ of the total irradiance. The irradiance values for the B 1 band filter from 455 - $515 \mathrm{~nm}, \mathrm{~B} 2$ band filter from 525 - $595 \mathrm{~nm}$, B3 band filter from $630-690 \mathrm{~nm}$, and B4 band filter from $763-898 \mathrm{~nm}$ are $1.20 \times 10^{2} \mathrm{~W} \mathrm{~m}^{-2}(9.44 \%), 1.31 \times 10^{2} \mathrm{~W} \mathrm{~m}^{-2}(10.32 \%)$, $9.49 \times 10^{1} \mathrm{~W} \mathrm{~m}^{-2}(7.47 \%)$, and $1.45 \times 10^{2} \mathrm{~W} \mathrm{~m}^{-2}(11.39 \%)$, respectively.

\subsection{Solar Irradiance at Short-Wave Infrared Band}

The FS5 RSI filter is designed specifically for different spectral bands in the visible and near infrared range. However, solar energy in the short-wave infrared range, from 1100 - $2500 \mathrm{~nm}$, can still pass through the filter, as shown in Figs. 7 - 11. This short-wave infrared range energy will reach the sensor detector and can be calculated using Eq. (9). The short-wave infrared energy of $2.19 \times 10^{2} \mathrm{~W} \mathrm{~m}^{-2}$ $(17.27 \%)$ will pass through the panchromatic filter, $2.10 \times$ $10^{2} \mathrm{~W} \mathrm{~m}^{-2}(16.52 \%)$ the $\mathrm{B} 1$ filter, $2.27 \times 10^{2} \mathrm{~W} \mathrm{~m}^{-2}(17.85 \%)$ the B2 filter, $2.08 \times 10^{2} \mathrm{~W} \mathrm{~m}^{-2}(16.38 \%)$ the $\mathrm{B} 3$ filter, and $2.06 \times 10^{2} \mathrm{~W} \mathrm{~m}^{-2}(16.45 \%)$ the B4 filter.

The solar irradiance shining into the RSI sensor can be calculated considering the filter effect. Therefore, the total solar energy passing through the panchromatic filter to reach the image sensor is composed of $35.08 \%$ from spectral range of $450-700 \mathrm{~nm}$ and $17.27 \%$ from the short-wave infrared region. The total becomes $9.13 \times 10^{4} \mathrm{~W} \mathrm{~m}^{-2}$ at the image sensor. Using the same approach, solar energy passing the $\mathrm{B} 1$ band filter is $4.53 \times 10^{4} \mathrm{~W} \mathrm{~m}^{-2}$, B2 band filter $4.91 \times 10^{4} \mathrm{~W} \mathrm{~m}^{-2}$, B3 band filter $4.16 \times 10^{4} \mathrm{~W} \mathrm{~m}^{-2}$, and B4 band filter $4.84 \times 10^{4} \mathrm{~W} \mathrm{~m}^{-2}$, respectively. Compared with the total solar energy of $1.744 \times 105 \mathrm{~W} \mathrm{~m}^{-2}$, note that $\sim 50 \%$ of the sun's radiance will reach the panchromatic sensor; $\sim 30 \%$ will reach the multi-spectral sensors. Table 1 summarizes the findings for both the panchromatic and multispectral sensor chips.

The FS5 CMOS sensor has been designed to panchromatic pixel size of $10 \mu \mathrm{m}$ and multi-spectral pixel size of $20 \mu \mathrm{m}$, as shown in Fig. 12. As a result, the worst case computed for the total sun exposure power on a PAN pixel is $9.13 \times 10^{-6} \mathrm{~W}$ and on a MS pixel is $2 \times 10^{-5} \mathrm{~W}$.

\subsection{Direct Sun Exposure Experiment}

To verify the above analyses and justify the FS5 RSI sensor integrity, a RSI sensor direct sun exposure was performed on the ground (Liu et al. 2012). Using a single lens with the F-number smaller than 6.4 was set up in front of the FS5 RSI sensor at noon to simulate direct sun exposure. A band-pass filter was placed in front of the sensor as shown in Fig. 13. The total solar power exposure was controlled using a power meter. The RSI sensor Signal Noise Ratio (SNR) values before and after the sun exposure were compared for both the PAN and MS sensors, as shown in Figs. 14 and 15, respectively. The tested image sensor was an engineering module with few bad pixels as indicated by the spikes in the plots. The consistent SNR values suggests that FS5 RSI sensor performance will not be influenced (or degraded) by direct sun exposure in orbit.

\section{CONCLUSION}

The potential maximum solar energy that might be exposed to the FS5 RSI CMOS sensor in orbit was investigated. The characteristics of the FS5 RSI band-pass filter and its effect on the sensor performance were also considered in this study. Sun exposure duration computed conservatively

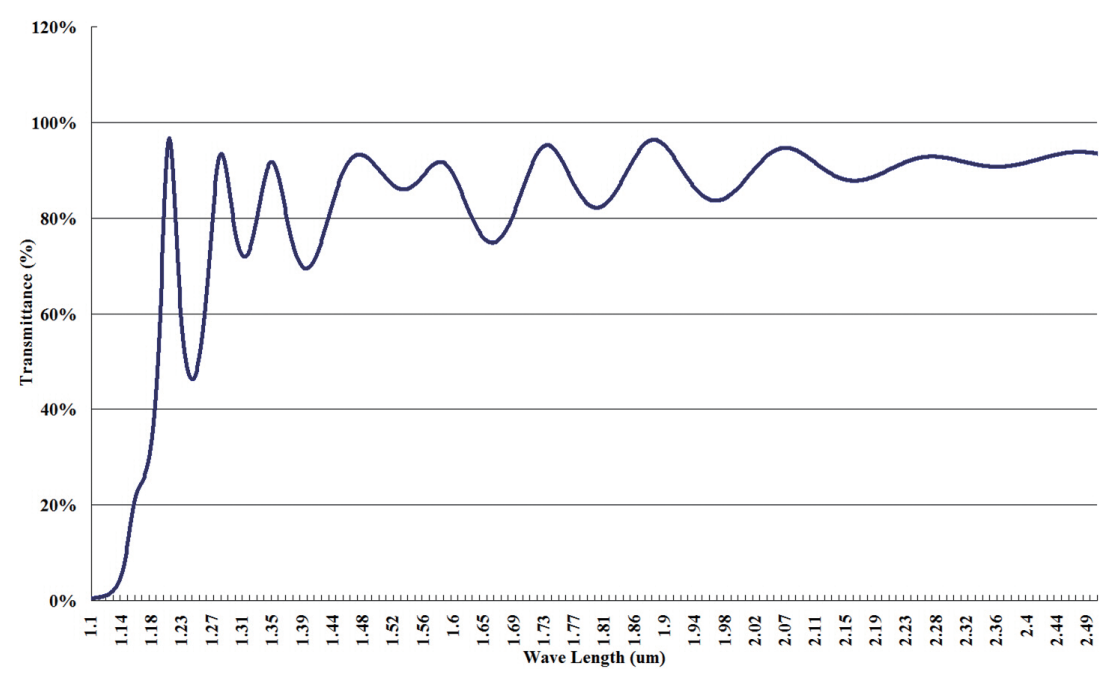

Fig. 7. Short-wave infrared transmittance profile of FS5 RSI panchromatic filter. (Color online only) 


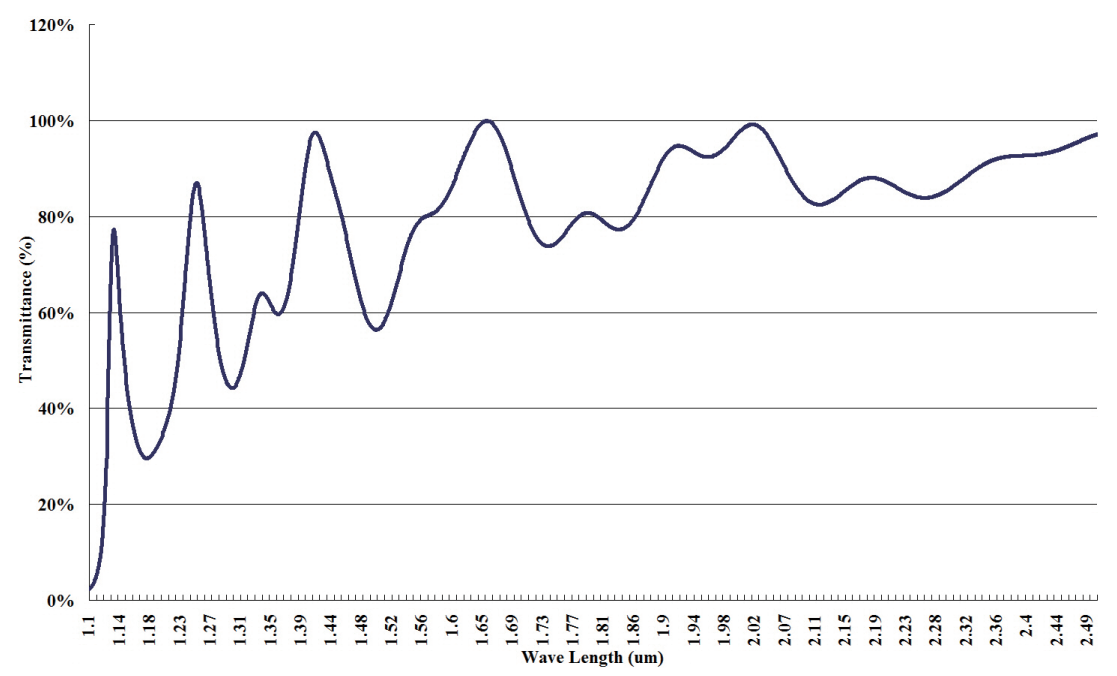

Fig. 8. Short-wave infrared transmittance profile of FS5 RSI B1 filter. (Color online only)

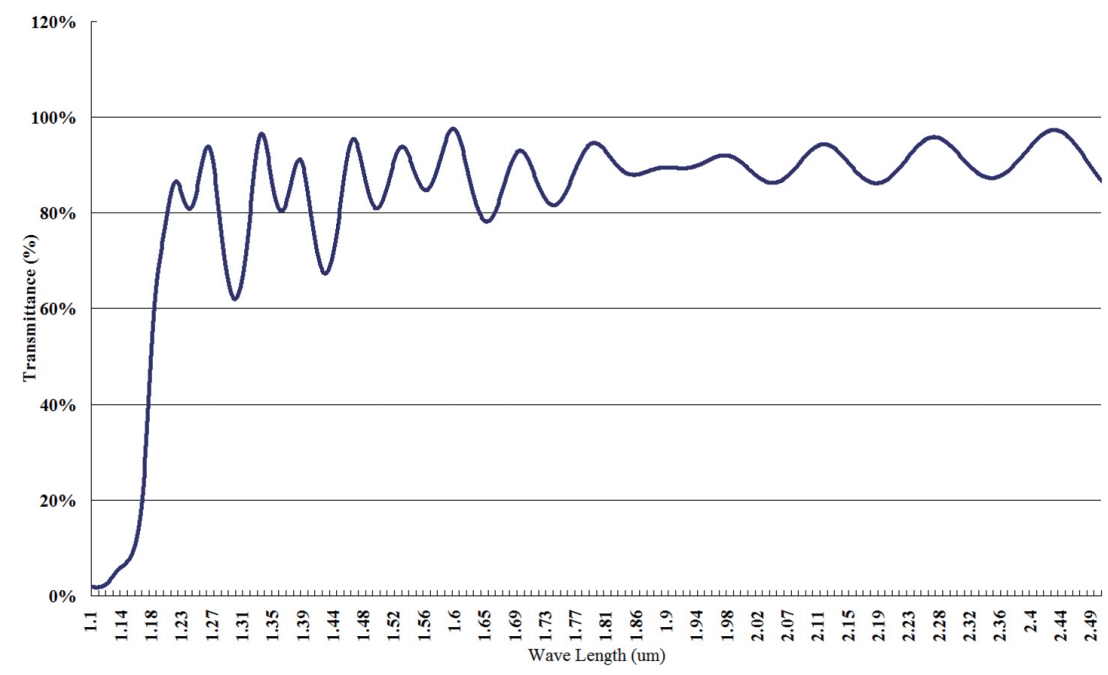

Fig. 9. Short-wave infrared transmittance profile of FS5 RSI B2 filter. (Color online only)

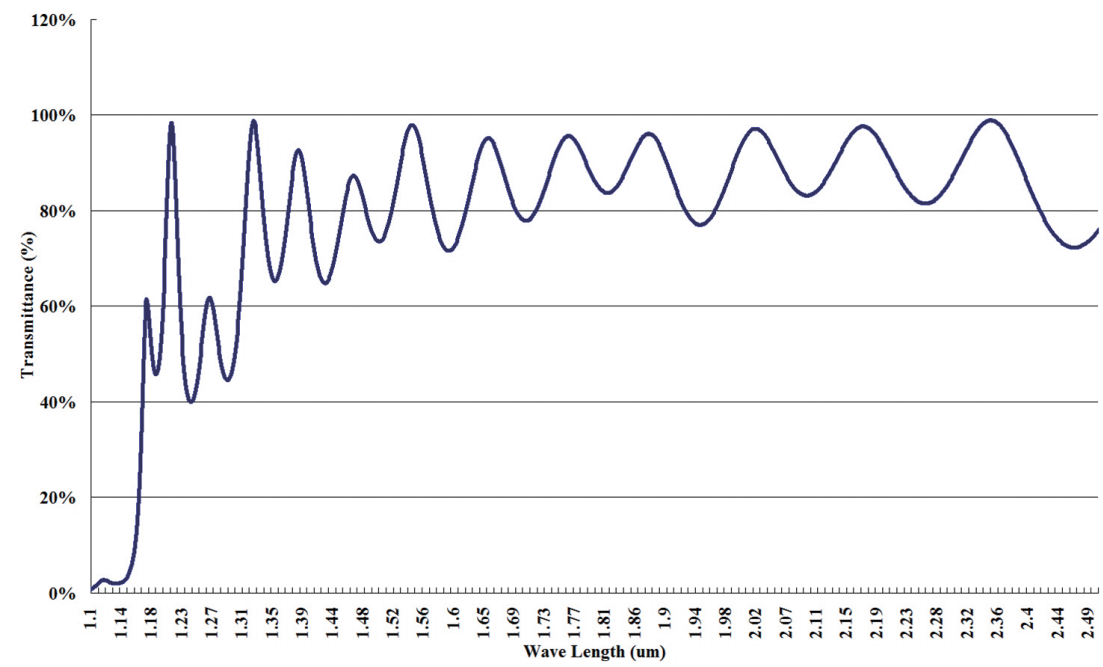

Fig. 10. Short-wave infrared transmittance profile of FS5 RSI B3 filter. (Color online only) 


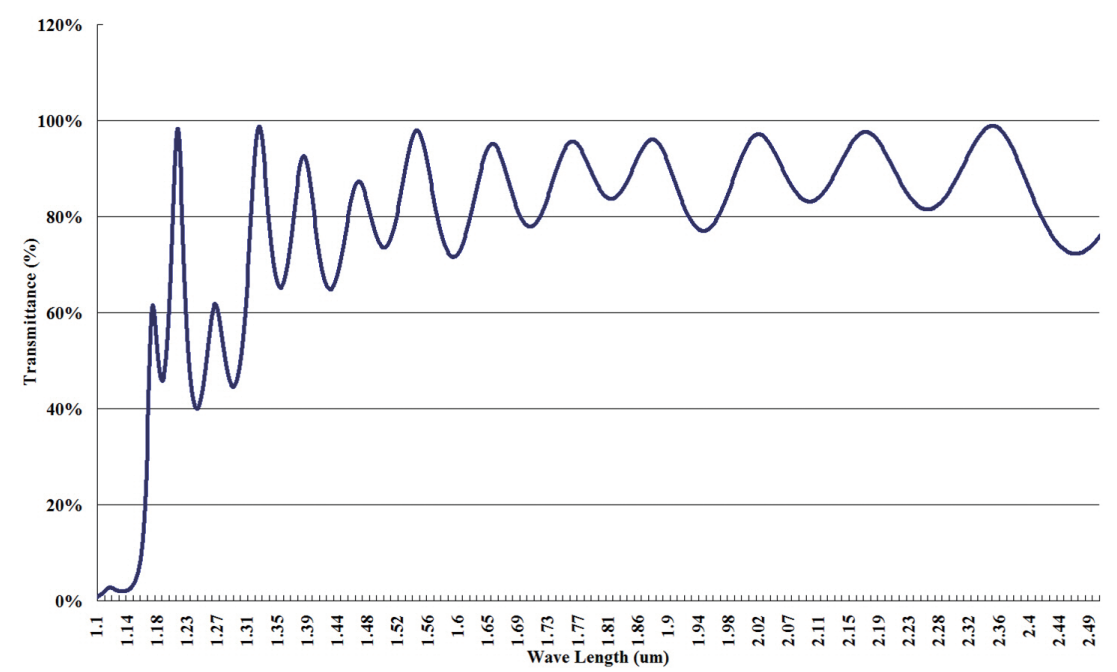

Fig. 11. Short-wave infrared transmittance profile of FS5 RSI B4 filter. (Color online only)

Table 1. Total irradiance for FS5 RSI both PAN and MS sensor chips.

\begin{tabular}{|c|c|c|c|}
\hline & Energy Percentage from visible to near-infrared range & Energy Percentage from short-wave infrared range & Total irradiance \\
\hline PAN & $35.08 \%$ & $17.27 \%$ & $9.13 \times 10^{4} \mathrm{~W} \mathrm{~m}^{-2}$ \\
\hline B1 & $9.44 \%$ & $16.52 \%$ & $4.53 \times 10^{4} \mathrm{~W} \mathrm{~m}^{-2}$ \\
\hline B2 & $10.32 \%$ & $17.85 \%$ & $4.91 \times 10^{4} \mathrm{~W} \mathrm{~m}^{-2}$ \\
\hline B3 & $7.47 \%$ & $16.38 \%$ & $4.16 \times 10^{4} \mathrm{~W} \mathrm{~m}^{-2}$ \\
\hline B4 & $11.39 \%$ & $16.45 \%$ & $4.84 \times 10^{4} \mathrm{~W} \mathrm{~m}^{-2}$ \\
\hline
\end{tabular}

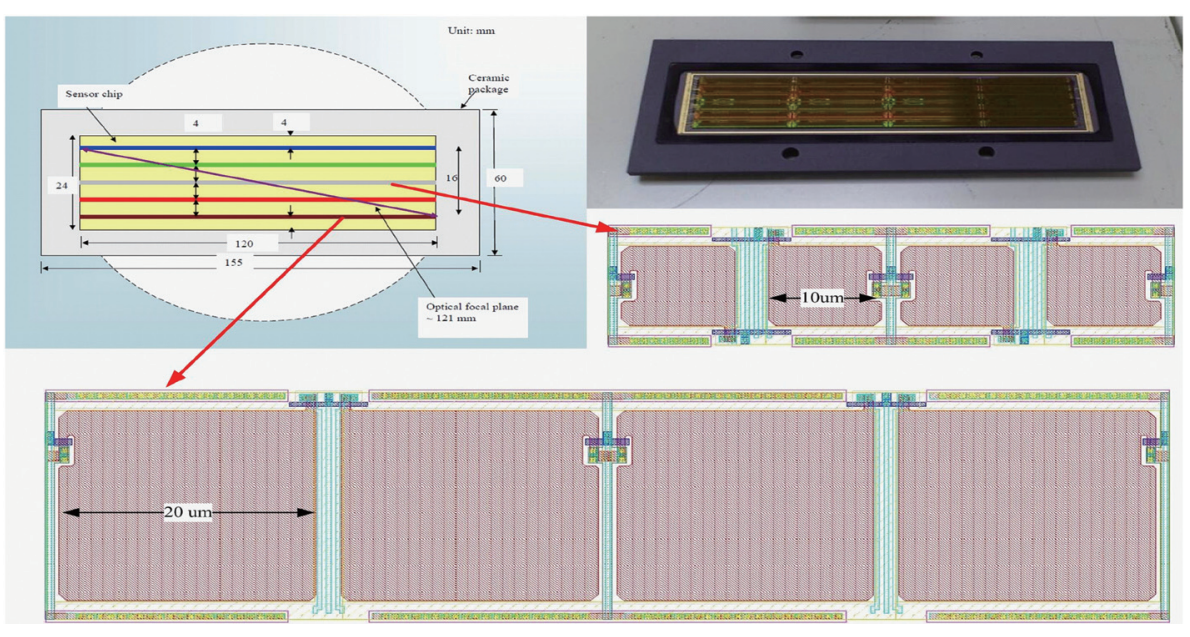

Fig. 12. The RSI image sensor. (Color online only)

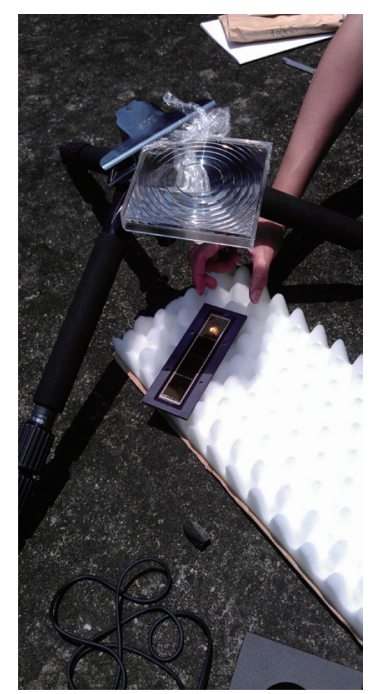

Fig. 13. Direct sun exposure test of RSI image sensor. (Color online only) 


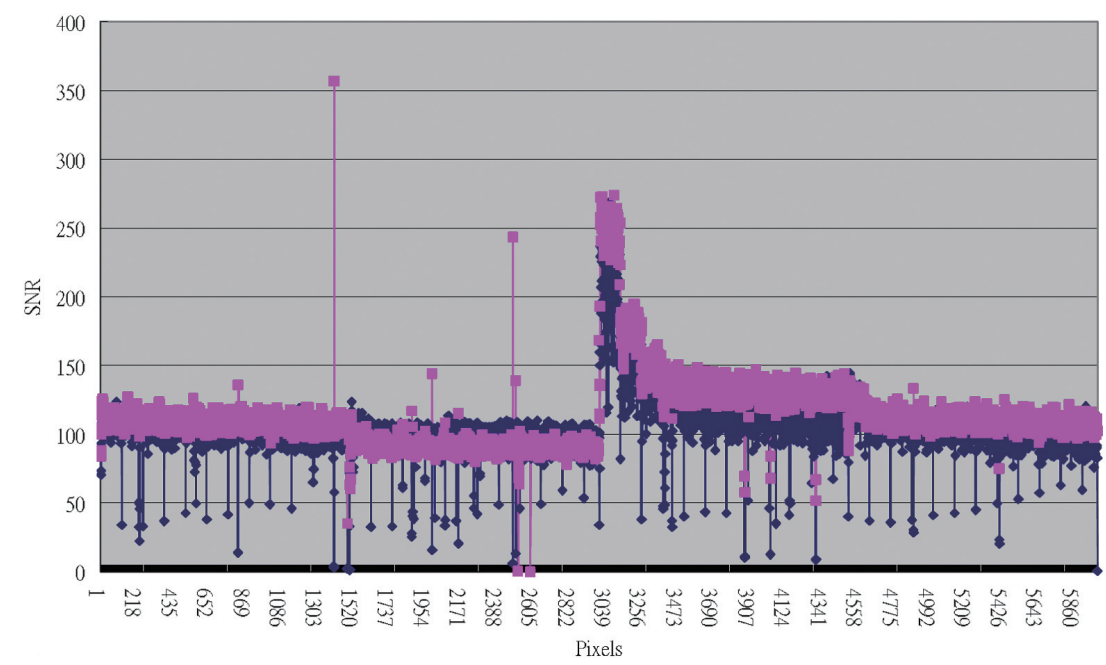

Fig. 14. SNR of PAN odd pixels, before (blue) and after (pink) sun exposure. (Color online only)

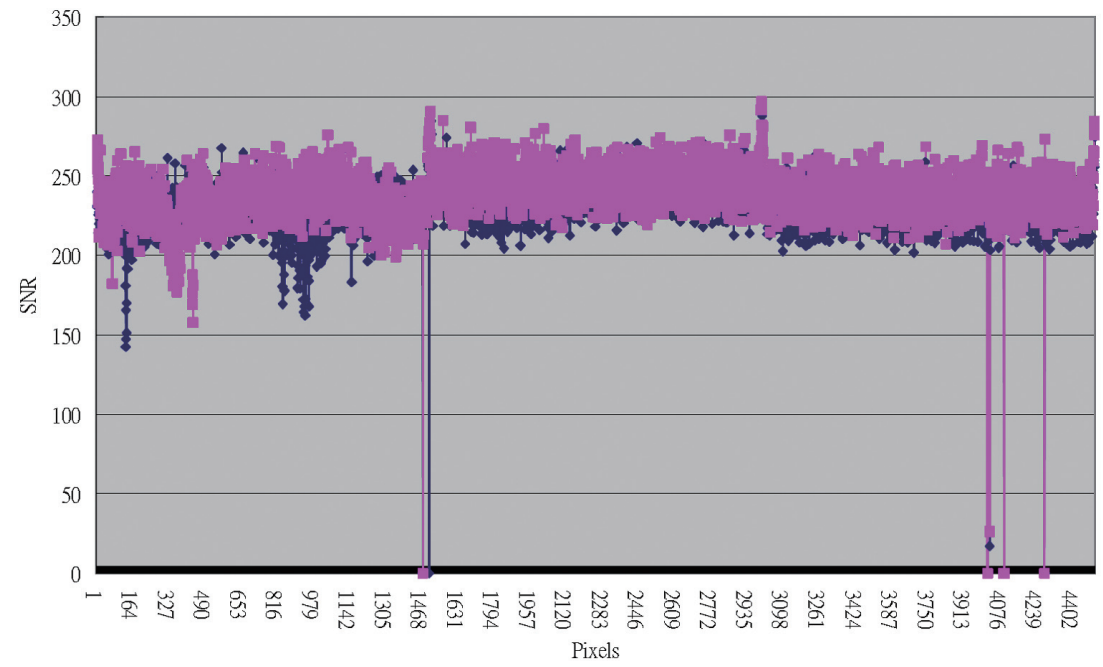

Fig. 15. SNR of B1 pixels, before (blue) and after (pink) sun exposure. (Color online only)

is driven mainly by the satellite tumbling speed right after separating from the launch vehicle. Direct sun exposure was performed on the ground to simulate a potential solar energy impact on the optical sensor performance in orbit. Based on both the analysis and experimental results, it is concluded that the FS5 RSI sensor can survive direct sun exposure during the ASH mode. In other words, FS5 RSI CMOS sensor performance will not be influenced (or degraded) by direct sun exposure in orbit.

Acknowledgements The presented study was supported by MOST project number 104-2221-E-492-026 and NSPO RSI project.

\section{REFERENCES}

Dereniak, E. L. and G. D. Boreman, 1996: Infrared Detec- tors and Systems, Wiley-Interscience, $592 \mathrm{pp}$.

Hsiao, C. N., H. P. Chen, P. K. Chiu, W. H. Cho, Y. W. Lin, and F.Z. Chen, 2010: Design and fabrication of optical thin films for remote sensing instruments. J. Vac. Sci. Tech., 28, 867-872, doi: 10.1116/1.3453700. [Link]

Irons, J. R., J. L. Dwyer, and J. A. Barsi, 2012: The next Landsat satellite: The Landsat Data Continuity Mission. Remote Sens. Environ., 122, 11-21, doi: 10.1016/j. rse.2011.08.026. [Link]

Liu, C., J. Lin, M. Y. Hsu, M. Y. Yeh, and C. H. Chang, 2012: RSI Sun exposure survivability. Proc. SPIE, 8516, doi: 10.1117/12.929220. [Link]

Palmer, J. M. and B. G. Grant, 2009: The Art of Radiometry, SPIE Publications, $384 \mathrm{pp}$.

Seabroke, G., A. Holland, and M. Cropper, 2008: Modelling radiation damage to ESA's Gaia satellite CCDs. Proc. SPIE, 7021, doi: 10.1117/12.790968. [Link] 